\title{
Educación Intercultural Bilingüe en el Ecuador
}

\author{
La propuesta educativa y su proceso
}

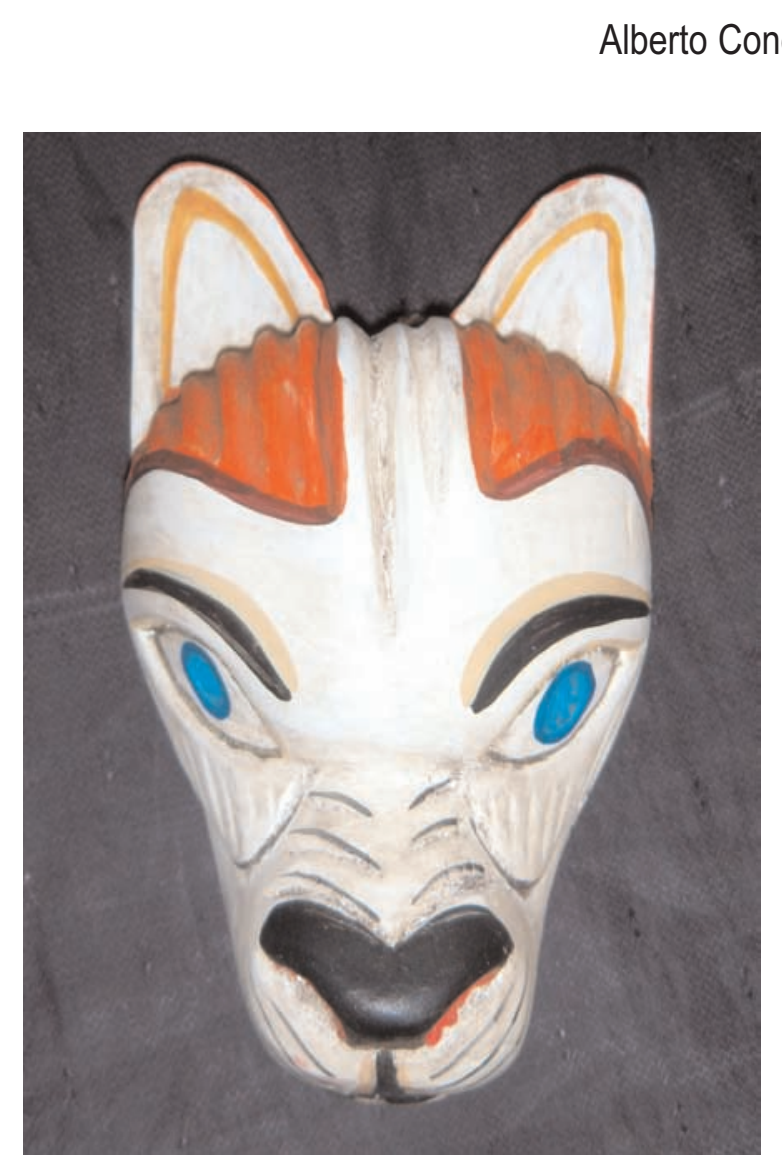

\section{Introducción}

El Ecuador es un país multilingüe, pluricultural, conformado por pueblos indígenas, población negra y población mestiza. Los pue- blos indígenas se encuentran en tres regiones del país: en la Costa, los awa, chachi, tsáchila y épera; en la Sierra los quichuas; en la Región Amazónica, los a'is (cofanes), sionas, secoyas, záparos, huaos, quichuas y los shuaras-achuaras, y mantienen una lengua y una cultura propia, que constituyen una de las riquezas culturales de la nación ecuatoriana.

Aun cuando la población ecuatoriana se caracteriza por esta enorme riqueza, la educación que se ha ofrecido a los pueblos indígenas ha estado orientada tradicionalmente a promover su asimilación indiscriminada, lo que ha contribuido a limitar su desarrollo socio-cultural y económico. Este tipo de educación, además, ha fomentado la ruptura de la identidad de los pueblos indígenas y el desarrollo de situaciones de racismo perjudiciales para el país.

La práctica de métodos memorísticos y repetitivos, el material didáctico empleado, y la misma organización de los establecimientos educativos han impedido el desarrollo de la creatividad y la participación de la población indígena en la vida nacional. A ello se suma el tipo de currículo en el que se ha basado el sistema que, debido a las características socio-culturales de la población indígena presenta limitaciones que

\footnotetext{
* Profesor de la Carrera de Educación Intercultural de la UPS y Supervisor Nacional de la DINEIB.
} 
dificultan y/o impiden cumplir con los objetivos educativos y lograr las metas esperadas.

Los maestros asignados a las comunidades indígenas desconocen, por lo general, la realidad de la población, al igual que su lengua y su cultura, siendo ello una causa para el mantenimiento de actitudes y comportamientos negativos que se traducen en el fomento de la desvalorización de la persona.

Los sistemas de evaluación y promoción que se mantienen influyen también de manera negativa en la sociedad, pues tienden a medir situaciones sin tener en cuenta lo que sucede en el campo. El parámetro de abandono escolar, por ejemplo, no puede tener las mismas consideraciones que en la ciudad debido a las actividades productivas que debe realizar la familia por las condiciones en que se encuentra.

Partiendo de la heterogeneidad de la población ecuatoriana, se considera indispensable definir una alternativa educativa que responda a la realidad socio-cultural de manera que se garantice una educación de óptima calidad, en la que se recupere y fortalezca el conocimiento y las prácticas sociales que los diferentes pueblos indígenas han logrado generar y mantener a través de su historia.

Frente a esta situación, en la última década se iniciaron una serie de experiencias educativas encaminadas a atender a la población indígena teniendo en cuenta sus características socioculturales y la capacidad de las lenguas indígenas para expresar todo tipo de conceptos sin necesidad de recurrir al castellano. Algunos de los proyectos basaron la metodología en el contexto de la etno-ciencia y de la integración de las distintas áreas del conocimiento a través de la aplicación de la teoría integrada de las ciencias.

Se considera que la modalidad escolar debe ser reemplazada por centros educativos comunitarios que recuperen el papel de la familia como responsable inmediata de la formación integral de la persona. Se considera, igualmente, que los responsables de estos centros debe ser personas con vocación, formación y preparación científica para orientar y guiar la educación de los miembros de la comunidad.

En consecuencia, el Gobierno Nacional establece como interés prioritario la atención educativa a los pueblos indígenas que, por mantener características sociales, culturales y lingüísticas particulares, requieren de la implementación de una política y de estrategias acordes con su realidad y las necesidades de desarrollo del país.

\section{Antecedentes}

La propuesta de Educación Intercultural Bilingüe tiene como referencia el resultado de las experiencias que se han desarrollado en el país en educación indígena en el transcurso de las últimas décadas, y junto con ello, las acciones legales desarrolladas para la ejecución de esta propuesta general de educación de la población indígena.

\section{Experiencias previas}

Las experiencias en educación indígena llevadas a cabo en el país han mantenido una orientación propia, y han alcanzado distintos grados de cobertura (local, regional y/o nacional). Todas ellas forman parte del desarrollo histórico de la educación bilingüe en el Ecuador.

Escuelas indígenas de Cayambe: En la década de los cuarenta se desarrolló una experiencia de educación indígena, una de cuyas maestras fue Dolores Cacuango que, con el apoyo de algunas mujeres quiteñas y de dirigentes indígenas de la zona, organizó un grupo de escuelas indígenas. Estas escuelas estuvieron ubicadas en Cayambe, provincia de Pichincha, y luego las misioneras Lauritas las extendieron hasta la provincia de Imbabura.

En estas escuelas trabajaron maestros indígenas de las mismas comunidades utilizando la lengua materna, revalorizando la cultura y 
la defensa de la tierra hasta que la última escuela dejó de funcionar con la Junta Militar, en 1963.

El Instituto Lingüístico de Verano (ILV): EL ILV, de procedencia estadounidense, inició sus labores en 1952 y las concluyó oficialmente en 1981. Su campo de acción estuvo localizado en algunas comunidades de las tres regiones del país, siendo su principal objetivo la evangelización y la traducción de la Biblia a las lenguas indígenas. La educación y otras actividades de atención a la población fueron realizadas en cuanto contribuían a facilitar el proceso evangelizador.

Para cumplir con sus objetivos llevó a cabo acciones de investigación lingüística, utilización de la lengua materna en la educación y formación de maestros indígenas. La política lingüística estuvo caracterizada por el mantenimiento de la escritura en base a dialectos de un mismo idioma o de la imposición del dialecto más prestigiado en otras zonas geográficas.

Misión Andina: Inició su labor en 1956, en la provincia de Chimborazo, con fondos provenientes de la Organización Internacional del Trabajo (OIT). Realizó acciones de desarrollo comunitario, educación, salud, asistencia agrícola y ganadera, formación artesanal, industria rural, ingeniería civil, servicios sociales, capacitación de personal.

En 1964, la Junta Militar nacionalizó la Misión Andina y le encargó la ejecución del programa de desarrollo rural del Plan Nacional de Desarrollo. Su jurisdicción se estableció en la Sierra en las áreas sobre los 1.500 metros de altura. En los inicios de la década de los setenta, la Misión fue integrada al Ministerio de Agricultura y luego desapareció. Se prepararon cartillas para lectura en lengua Kichwa sobre temas que incluyen mitología y aspectos sociales y otros relacionados con la naturaleza, Este trabajo se realizó empleando los dialectos locales de Salasaca, Imbabura, Chimborazo.
Escuelas Radiofónicas Populares del Ecuador (ERPE): Estas escuelas, dirigidas a la alfabetización de la población adulta de habla kichwa, surgieron en 1964 por iniciativa de Monseñor Proaño, Obispo de Riobamba. Aunque tenían alcance para la Sierra, la labor se centró en la provincia de Chimborazo y Tabacundo, en la provincia de Pichincha.

La lengua materna tuvo como objetivo la concientización antes que la utilización en la educación. Actualmente sólo transmiten en español.

Sistema Radiofónico Shuar (SERBISH): Desde 1972 funcionan las escuelas radiofónicas de los Shuar-achuar que iniciaron con la primaria y luego fueron extendiéndose hasta incluir la secundaria. Actualmente cuentan también con un Instituto Pedagógico Intercultural Bilingüe que surgió a partir del Instituto Normal Bilingüe Intercultural, y están iniciando la introducción de la modalidad presencial con cobertura en las provincias de la Región Amazónica y algunos sectores de migrantes en la Costa.

En 1979 se oficializó el Sistema de Educación Radiofónico Bilingüe Bicultural Shuar que funciona con el auspicio de la Misión Salesiana, la Federación Interprovincial de Centros Shuar y Achuar y el Ministerio de Educación.

Emplean la lengua materna y el español en materiales para la primaria y secundaria producidos en los dos idiomas, aunque los contenidos se han centrado en la traducción de los de la educación tradicional en español. Uno de los aspectos importantes ha sido la formación de maestros indígenas y auxiliares radiofónicos.

Escuelas Indígenas de Simiatug: Estas escuelas funcionan dentro de la organización indígena «Fundación Runacunapac Yachana Huasi", cuyo campo de acción es la Parroquia Simiatug, en la provincia de Bolívar. Tienen como entidades de apoyo el "Instituto Simiatuccunapac Jatun Capari y la emisora de la 
Fundación. Las actividades educativas de las escuelas se adecuan a las labores de Instituto dado que los maestros estudian en ese centro.

Este proyecto ha elaborado un texto para alfabetización de los niños utilizando el sistema kichwa de escritura unificada. La dificultad que enfrentan es la existencia de niños que no han adquirido el kichwa como lengua materna por lo que se requiere formar maestros para enfrentar esta situación.

Sistema de Escuelas Indígenas de Cotopaxi (SEIC): Este programa se inició en 1974 bajo el auspicio de religiosos salesianos del grupo de pastoral de Zumbahua y Chucchilán con cobertura extendida en diversas comunidades a más de la provincia de Cotopaxi. Actualmente utilizan la lengua materna como lengua principal de educación y han formado maestros de las propias comunidades. También han organizado proyectos productivos vinculados a la educación. Para el nivel medio cuentan con el colegio «Jatari Unancha» de modalidad semi-presencial.

Escuelas bilingües de la Federación de comunas "Unión de Nativos de la Amazonía Ecuatoriana (FCUNAE): Comenzaron con unas pocas escuelas, en 1975, para extenderse luego a unas cincuenta comunas de la Federación. Realizaron algunas investigaciones en el campo de la historia, produjeron material didáctico en lengua kichwa para niños y formaron maestros de las propias comunidades. Este proyecto desapareció por falta de apoyo de algunos dirigentes de la organización local.

Subprograma de alfabetización kichwa: El programa inició en 1978, en el Instituto de Lenguas y Lingüística de la PUCE, y funcionó entre 1979 y 1986 bajo la responsabilidad del "Centro de Investigaciones para la Educación Indígena" (CIEI). En 1980 se desarrolló el Modelo Educativo "Macac" y se elaboró material para alfabetización en lenguas kichwa, secoyasiona, huao y chachi; se produjo material para

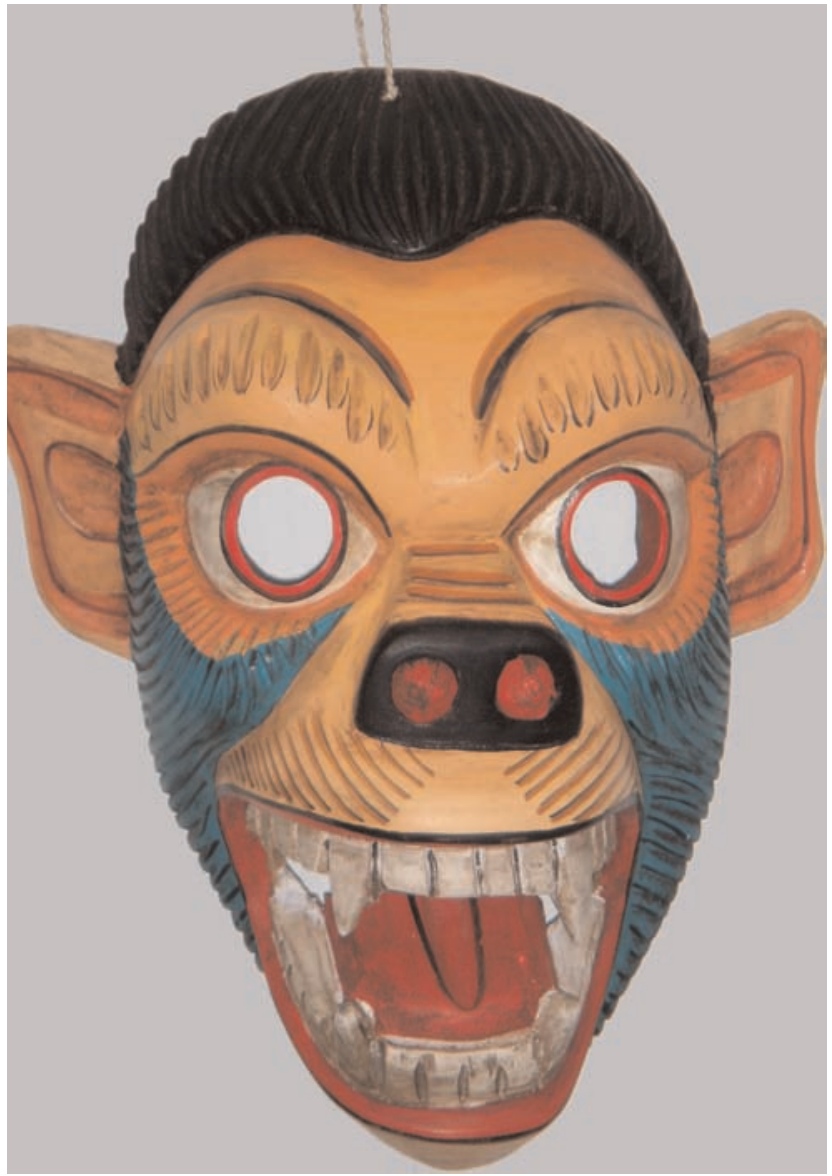

post-alfabetización, educación de niños y enseñanza de castellano como segunda lengua para niños y adultos kichwa hablantes.

En esta etapa se inició el proceso de unificación del sistema de escritura del kichwa. Se utilizó la lengua materna como lengua principal de educación y el español como lengua de relación intercultural, y se formaron gran parte de los futuros líderes de las comunidades indígenas. Los educadores fueron indígenas de las mismas comunidades. De todos los proyectos de educación bilingüe desarrollados hasta 1988, es el de mayor cobertura ya que el programa tuvo un carácter nacional.

Como un antecedente a este programa se tiene la licenciatura en lingüística kichwa que funcionó entre 1974 y 1984, en el Instituto de 
Lenguas y Lingüística de la Universidad Católica, en donde se formó un significativo número de indígenas.

Chimborazoca Caipimi: Paralelamente al subprograma de alfabetización kichwa, dirigido por el CIEI, existió un proyecto exclusivo para la provincia de Chimborazo. En este proyecto se preparó una cartilla, además de algunos materiales de lectura para adultos. En este proyecto se utilizó la escritura del habla local.

Colegio Nacional "Macac": Inició el programa de auto-educación bilingüe intercultural en lengua kichwa en 1986 como parte de las actividades de la Corporación Educativa "Macac". Atiende el nivel de educación secundaria con la formación de prácticos y bachilleres técnicos. Mantiene asesoramiento permanente a la escuela bilingüe "Atahualpa" de la comunidad Chaupiloma, en la provincia de Pichincha.

A más de la participación de los estudiantes del programa, cuenta con la presencia de miembros de las comunidades a través del programa de educación desescolarizada sin distinción de niveles de educación, conocimientos, edad, etc. Tiene cobertura nacional, y constituye una continuación del Modelo Educativo «Macac»; emplea la lengua kichwa como lengua principal de educación y el español como segunda lengua; utiliza el sistema de escritura unificada, e integra la producción al proceso educativo.

La Corporación realiza, para el colegio "Macac", investigaciones que se reinvierten en la corrección permanente del proceso educativo, en material didáctico, en la formación personal de los participantes, mediante reuniones destinadas a fomentar la revalorización cultural y psicológica, a más de profundizar el conocimiento científico.

Proyecto de Educación Bilingüe Intercultural: Inició su labor en 1986 con la firma de un convenio entre la GTZ, organismo de la República Federal de Alemania, y el Gobierno ecuatoriano. Han trabajado en la elaboración de una propuesta curricular, material didáctico de educación primaria kichwa, capacitación y apoyo a las organizaciones indígenas en el campo de la promoción educativa y cultural. $\mathrm{Ha}$ empleado el sistema de escritura unificada. Atiende 53 escuelas en siete provincias de la Sierra del país y ha participado, conjuntamente con la Universidad de Cuenca, en la capacitación de dos promociones de estudiantes, en la carrera de educación bilingüe a nivel de licenciatura.

Proyecto Alternativo de educación Bilingüe de la CONFENIAE (PAEBIC): Funciona desde 1986, ha trabajado en ocho escuelas de las provincias de Napo y Pastaza. Ha elaborado material educativo para los primeros grados de la primaria. Emplea el sistema de escritura unificada.

Convenio entre el MEC y la Confederación de Nacionalidades Indígenas del Ecuador (CONAIE): La CONAIE, con la participación de sus organizaciones miembros, preparó la propuesta educativa para el Gobierno Nacional, lo que dio como resultado la creación de la Dirección Nacional de Educación Intercultural Bilingüe (DINEIB), en noviembre de 1988.

En 1989 se firmó un convenio de Cooperación Científica entre el MEC y la CONAIE con el propósito de realizar investigaciones lingüísticas y pedagógicas, así como para elaborar material didáctico de alfabetización, post-alfabetización y de formación progresiva para el personal que participa en la educación en lenguas kichwa, awa, chachi, tsachila y las otras existentes en el país. Para mejorar la situación pedagógica, la CONAIE ha iniciado la conformación de un equipo para elaborar material sobre diferentes aspectos de la educación intercultural bilingüe.

Convenio entre el MEC y la Federación Nacional de Indígenas Evangélicos: Este convenio fue suscrito en el año 1990, con el fin de realizar investigaciones destinadas a producir 
material didáctico para la enseñanza de ciencias sociales y de español como segunda lengua.

\section{Marco jurídico de la EIB}

Las bases legales directas sobre las que se cimenta la educación intercultural bilingüe -aparte de las que establece la Ley de Educación, la Ley de Cultura, y sus reglamentos-, son las siguientes:

En 1983 se reformó el Art. 27 de la Constitución de la República que determina que "En los sistemas de educación que se desarrollan en las zonas de predominante población indígena, se utilice como lengua principal de educación el kichwa o la lengua de la cultura respectiva y el castellano como lengua de relación intercultural".

El 12 de enero de 1982 se promulgó el Acuerdo Ministerial 000529 mediante el cual se acordó: "Oficializar la educación bilingüe bicultural, estableciendo en las zonas de predominante población indígena planteles primarios y medios donde se imparta instrucción en los idiomas kichwa y castellano o su lengua vernácula”.

El 15 de noviembre de 1988, mediante Decreto Ejecutivo 203 que reforma el Reglamento General a la Ley de Educación, se institucionaliza la educación intercultural bilingüe, con la creación de la Dirección Nacional de Educación Indígena Intercultural Bilingüe (DINEIB) con funciones y atribuciones propias entre las que se pueden mencionar las siguientes:

- desarrollar un currículo apropiado para cada uno de los subsistemas y modalidades de educación intercultural bilingüe;

- diseñar modalidades educativas acordes con las necesidades de la población indígena;

- promover la producción y utilización de materiales didácticos de acuerdo con los criterios lingüísticos pedagógicos y sociales adecuados;
- velar por la aplicación de una política lingüística que tome en cuenta un sistema de escritura unificada para cada lengua basado, en lo posible, en el criterio fonológico;

- planificar, dirigir y ejecutar la educación intercultural bilingüe, en coordinación con el CONADE y con las organizaciones de las nacionalidades indígenas del Ecuador;

- proponer al nivel directivo superior proyectos, instrumentos legales y reglamentarios y modificar los vigentes, para alcanzar una acción educativa eficiente;

- dirigir, orientar, controlar y evaluar el proceso educativo en todos los niveles, tipos y modalidades de la educación intercultural bilingüe. Las organizaciones participarán según su representatividad;

- coordinar con las demás direcciones nacionales las informaciones provenientes de las direcciones provinciales interculturales bilingües relativas a la implementación y ejecución del currículo, las investigaciones y evaluaciones relacionadas a la educación intercultural;

- desarrollar, implementar y evaluar las acciones de educación para la salud conservación del medio ambiente y bienestar estudiantil;

- desarrollar, implementar y evaluar programas y proyectos educativos para el desarrollo de las comunidades indígenas;

- organizar los establecimientos de educación intercultural bilingüe en los ni veles preprimario, primario y medio;

- formar y capacitar profesores y demás recursos básicos para la educación intercultural en los diferentes lugares del país;

- dirigir, organizar y orientar la educación de los institutos pedagógicos bilingües.

En el año 1992, el Congreso Nacional reforma la Ley de Educación mediante la cual se reconoce la educación intercultural bilingüe en 


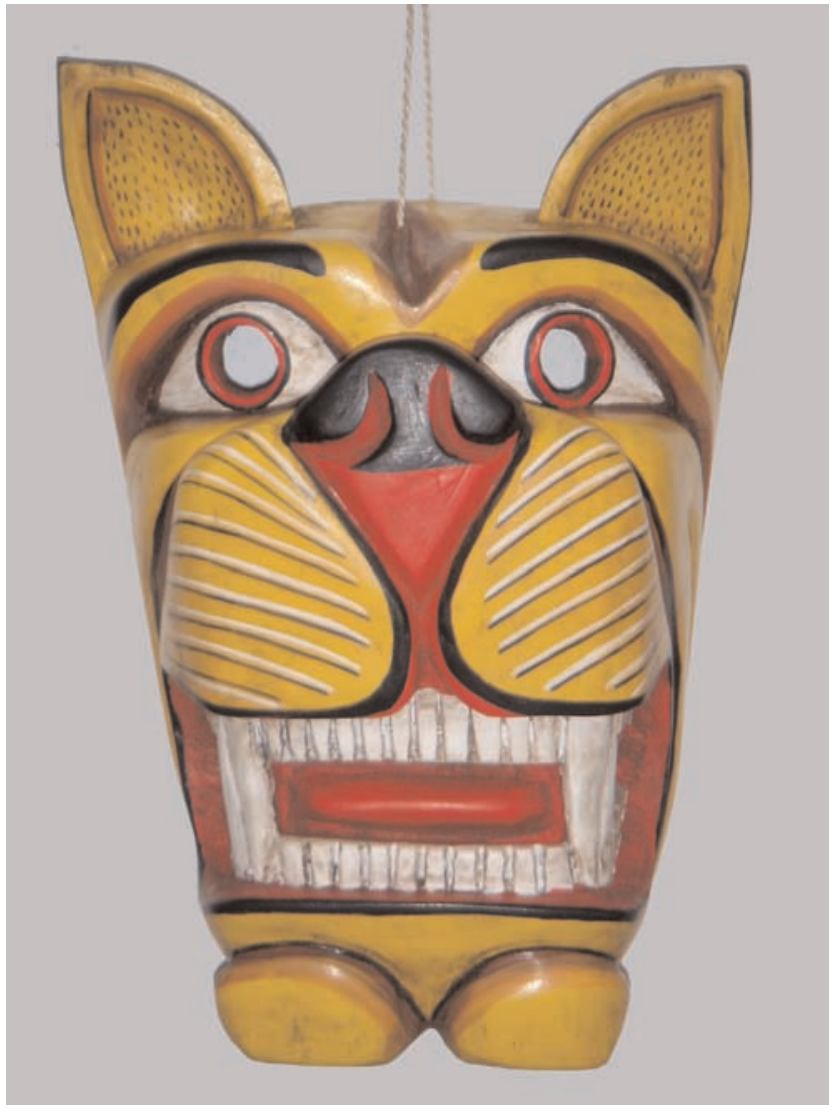

el marco de la Ley, y se reconoce a la DINEIB (Dirección Nacional de Educación de Educación Intercultural Bilingüe) la autonomía técnica, administrativa y financiera.

La vigencia del convenio 169 de la Organización Internacional de Trabajadores OIT.

El Modelo de Educación Intercultural Bilingüe (MOSEIB) que se expidió a través del Registro Oficial N. 278 de 17 septiembre del 1993.

\section{Justificación}

La realidad multicultural y plurilingüística del Ecuador tiene como antecedentes inmediatos la creación de la DINEIB, encargada de implementar la educación intercultural para atender las necesidades educativas de los pueblos indígenas.
La crisis general bajo la cual se desarrolla la educación en el país, al igual que lo que sucede en otros lugares del mundo, obliga a emprender una profunda revisión de las acciones educativas a fin de encontrar soluciones frente a la pérdida de recursos que se invierten sin lograr los efectos deseados.

Como parte de la crisis se encuentra que la persona ha dejado de ser el centro del quehacer educativo y que, como consecuencia de ello, aspectos tales como la formación de la persona, la atención sicológica que requiere la población estudiantil, el desarrollo de la creatividad y el fortalecimiento de la identidad cultural, entre otros, han sido sustituidos por una excesiva preocupación sobre aspectos formales tales como la constante reelaboración de planes de programas y la definición del currículo; la insistencia en el cumplimiento de regulaciones que por lo general no son aplicables, etc.

Junto con la falta de preocupación por la persona, se encuentra que la familia ha descargado gran parte de su responsabilidad en el sistema escolar; ello ha dado lugar a que éste se haya subrogado una serie de responsabilidades que no puede cumplir por no tener capacidad para responder a las necesidades de la población estudiantil ni a las nuevas expectativas de los padres de familia. Bajo estas circunstancias, la familia ha quedado al margen de la educación de sus hijos, convirtiéndose la escuela en un ente aislado sin ninguna relación con los grupos sociales que le rodean, es decir, la familia y la comunidad.

Con respecto a las regulaciones administrativas, se encuentra que existe una excesiva rigidez debido a la sobrevaloración de las normas y a la minusvalorización de la persona. Esta situación impide el desarrollo de la persona, tanto en lo que se refiere a sus capacidades individuales como a la adquisición de conocimientos que le enriquezcan y le preparen para actuar con solvencia en la vida.

Se encuentra que el sistema adolece de fallas como las siguientes: 
- empleo de métodos memorísticos y repetitivos;

- acumulación de conocimientos distribuidos en materias o asignaturas que muchas veces no tienen utilidad ni presentan interés por ser obsoletos y poco útiles;

- exigencia de aprender determinadas teorías, como es el caso de la matemática y de la gramática que descuidan la enseñanza de los sistemas y procesos lógicos y del uso y desarrollo de la capacidad expresiva de la lengua;

- masificación y uniformidad en la administración de conocimientos sin atender los intereses ni las capacidades personales;

- ausencia de relación entre los distintos niveles educativos;

- métodos de evaluación basados en objetivos punitivos más que en instrumentos orientadores para atender las necesidades de los estudiantes;

- mantenimiento de parámetros negativos de promoción (repetición, deserción, abandono, etc.);

- despersonalización que se deriva de las prácticas formales mal aplicadas como son las de "correr lista", formarse, mantener horarios y calendarios inamovibles y otras similares;

- Falta de formación, preparación y dedicación de una gran parte de maestros y administradores del sistema que, en el ejercicio de sus funciones, anteponen otros intereses a los de tipo educativo.

Estas situaciones se traducen en el mantenimiento de un tipo de currículum inadecuado que poco favorece el desarrollo de la persona para llegar a una plena realización. Se evidencia que el sistema mantenido no ha incidido en un cambio significativo ni el mejoramiento de la calidad y condiciones de vida de la población. Por el contrario, se ha favorecido el abandono del campo y la migración, así como el deterioro de la vida en las comunidades.
Con respecto al desarrollo intelectual y de la creatividad, y a las posibilidades de acceso al conocimiento, se encuentran importantes carencias. El fortalecimiento de los valores culturales propios de las comunidades, por su parte, ha sufrido un notable deterioro a causa de la imposición de seudo-valores introducidos a través del propio sistema educativo y del mal uso de los medios de comunicación.

La falta de oportunidades para el desarrollo de la socialización que, entre otras cosas, debe ser función de la escuela y más instituciones educativas, ha sido una de las causas que ha contribuido a la escasa participación de la población en la vida política del país.

En el caso de la población indígena, la situación educativa resulta mucho más conflictiva puesto que se suman otros condicionamientos como son el problema cultural, la lengua de educación, las condiciones socio-económicas, la carencia de personal formado para la docencia y la administración del sistema escolar, la falta de material didáctico, la imposición de horarios y calendarios inadecuados, etc. Aparte de ello, se reconoce la influencia del sistema tradicional de habla hispana que, en la práctica, no ha podido ser modificado o acondicionado a las necesidades de la población indígena como eran los planteamientos iniciales.

Determinadas comunidades indígenas, por influencia de profesores y autoridades locales, no reconocen ni el bilingüismo ni los conocimientos tradicionales de las culturas indígenas como valores reales y vigentes. Como una consecuencia de ello, han adoptado posiciones desfavorables frente a la nueva alternativa de educación.

Debido a las condiciones de sometimiento y dominación a las que ha estado sujeta la población de manera tradicional, se constata la necesidad de que el sistema educativo contribuya a la solución de problemas relacionados con la formación de la persona en toda su integridad incluyendo el respeto a su identidad cultural. 
Junto con ello se considera necesario recuperar el rol de la familia en la educación dado que, debido a las influencias de acciones provenientes de ámbitos externos, se han introducido sistemas que, en lugar de fortalecer los lazos familiares, tienden a provocar la desintegración de la familia y a ahondar la brecha generacional.

Se constata también que el sistema educativo actual promueve, en gran medida, la integración indiscriminada de la población indígena a la sociedad mestiza a través de la creación de una imagen falsa con respecto a la absoluta validez de la cultura occidental, situación que se produce por la desvalorización y/o negación de los contenidos culturales y prácticas provenientes de las sociedades indígenas.

Se evidencia una práctica generalizada de actitudes neocolonialistas que tienden a la eliminación del conocimiento como instrumento de desarrollo y de solución de problemas socio-culturales y económicos existentes.

La presencia de la institución escolar en las comunidades representa, por lo general, un

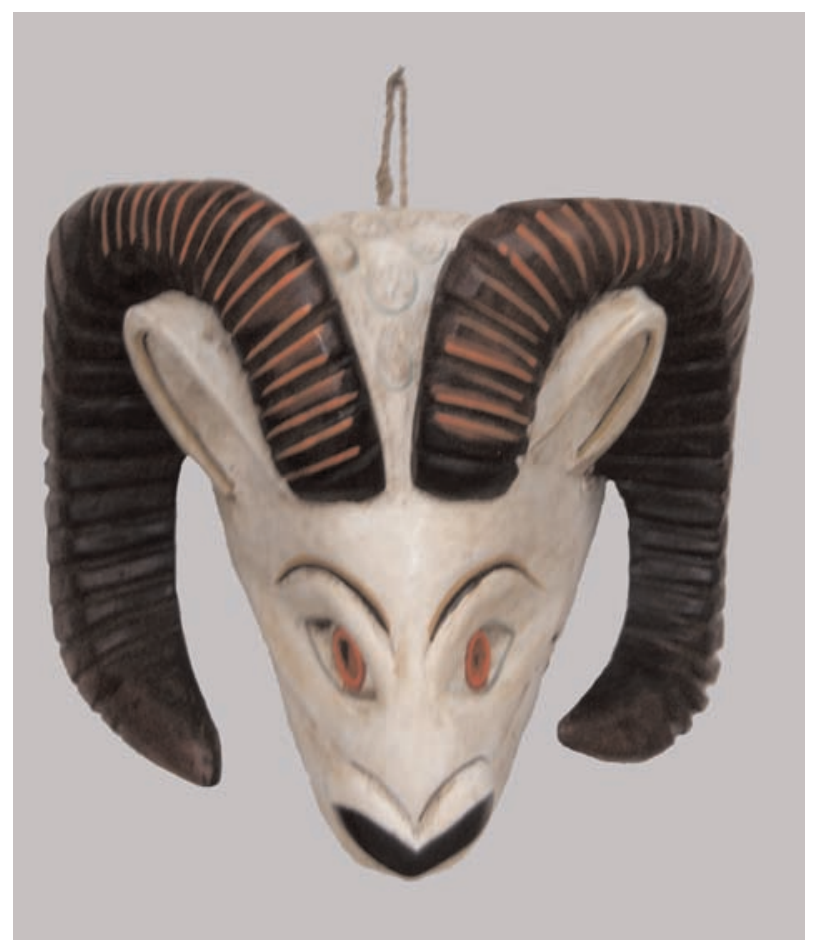

elemento disociador por la ausencia de relación con las organizaciones locales. En este contexto, el maestro -al igual que otros agentes externos-, detentan un poder adicional que en lugar de favorecer a la comunidad representa un factor de división por la serie de grupos que tienden a crearse como son, por ejemplo, las asociaciones de padres de familia, los clubes deportivos, los grupos de mujeres para atender determinadas demandas de la escuela, etc. Instituciones no tradicionales, como las mencionadas, deben tener en cuenta las características propias de la cultura respectiva con una limitada influencia del personal escolar.

La necesidad de que la institución educativa se relacione con la comunidad y de que sea parte de ella como un organismo más del sistema interno, constituye una de las necesidades más urgentes.

Debido, entre otras causas, a la tradición establecida, y a la ausencia de referentes que permitan optar por otras alternativas de educación, gran parte de la población indígena se ha habituado a creer que el único medio válido para mejorar sus condiciones es a través de la adopción del castellano y del abandono de la lengua nativa, aun cuando el sistema no se preocupa de enseñar la lengua española a la población que habla un idioma materno diferente.

En la práctica se observa que la institución escolar no ha hecho esfuerzos suficientes para resolver la situación de los niños indígenas que tienen que competir con estudiantes de habla hispana en un sistema que responde a situaciones, por lo general, citadinas. Bajo estas condiciones, la población aprende a minusvalorar su lengua nativa y a despreciar la cultura con la consiguiente pérdida de identidad, y con el agravante de que el tipo de castellano que le sirve de modelo es, por lo general, causa de discriminación por la pobreza de vocabulario, el uso de palabras con significados semántica y socialmente erróneos, y la sintaxis defectuosa. 
La falsa creencia de que las lenguas indígenas no pueden expresar una diversidad de conceptos, así como la actitud negativa que mantiene la sociedad dominante frente a estas lenguas, contribuye a acentuar las dificultades de aprendizaje, a fomentar una actitud de minusvaloración y a mantener las condiciones históricas de opresión, lo que obliga a tomar medidas que contrarresten esta situación.

La realidad multicultural que caracteriza a la sociedad ecuatoriana y que ha sido tradicionalmente ignorada por los grupos dominantes ha conducido a crear situaciones de aislamiento y marginación perjudiciales para las comunidades y para el país. Los pueblos indígenas se han encontrado impedidos de acceder a conocimientos, tecnologías y avances científicos por la imposibilidad de contar con información. Muchos conocimientos son actualmente compartidos por infinidad de pueblos en el mundo, sin que por ello se hayan producido, necesariamente, procesos negativos de aculturación o deculturación, pues cada sociedad sabe cómo integrarlos cuando dispone de los medios informativos suficientes.

A pesar de los esfuerzos realizados en los últimos años para atender las necesidades educativas de la población indígena, se encuentra que existen vacíos que hace falta llenar a fin de proporcionar una educación eficiente.

La implementación del sistema de educación intercultural bilingüe requería, con anterioridad a la promulgación del acuerdo de creación de la DINEIIB, contar con un proceso de planificación y organización que definiera todos los aspectos de orden legal, administrativo y curricular necesarios para lograr la eficiencia esperada por las organizaciones y la población.

En la actualidad se requiere definir una serie de aspectos tendientes a corregir los errores del pasado a fin de responder a las expectativas y necesidades de la población indígena y del país, a través de la reorientación del proceso como se propone en el presente documento.

\section{Visión de la DINEIB}

"La Dirección Nacional de Educación Intercultural Bilingüe, especialista en el desarrollo de las lenguas y culturas indígenas, es una institución pública protagonista en la gestión de procesos educativos en lenguas indígenas y castellano como segunda lengua; con personal intercultural bilingüe optimizado que responda con responsabilidad al servicio de la comunidad". 1

\section{Misión de la DINEIB}

"Emprender el desarrollo de las políticas de la educación intercultural bilingüe, como políticas públicas con la participación organizada de los actores sociales contemplados en el modelo educativo intercultural bilingüe; dinamiza las iniciativas para la construcción de una sociedad intercultural, impulsa proyectos de mejoramiento de las condiciones y calidad de vida de las comunidades, y promueve el fortalecimiento de la identidad cultural y los procesos organizativos de los pueblos y nacionalidades".2

\section{Principios de la Educación Intercultural Bilingüe}

Los principios que sustentan el modelo de educación intercultural bilingüe son los siguientes:

- el eje principal del proceso educativo es la persona a cuyo servicio debe estar el sistema de educación;

- la familia representa la base del proceso de formación de la persona y es la principal responsable de su educación;

- la comunidad y la organización comunitaria son corresponsables, junto con el Estado, de la formación y educación de sus miembros;

- la lengua nativa constituye la lengua principal de educación, y el español tiene el rol 
de segunda lengua y lengua de relación intercultural;

- tanto la lengua nativa como el español deben expresar los contenidos propios de la cultura respectiva;

- los conocimientos y prácticas sociales de los pueblos indígenas son parte integrante del sistema de educación intercultural bilingüe;

- el sistema de educación intercultural bilingüe debe fomentar la recuperación de la calidad de vida de la población en todos sus aspectos;

- la educación dirigida a la población indígena debe proporcionarle la mayor información posible, y por todos los medios de comunicación a su alcance, para facilitarle acceso al conocimiento;

- el currículo debe tener en cuenta las características socio-culturales de las culturas correspondientes y los avances científicos logrados en este campo en diversas experiencias realizadas en el país;

- el currículo debe integrar los aspectos psicológicos, culturales, académicos y sociales en función de las necesidades de los estudiantes.

\section{Fines}

Son fines de la Educación Intercultural bilingüe los que se indican a continuación:

- Apoyar el fortalecimiento de la interculturalidad de la sociedad ecuatoriana;

- Fortalecer la identidad cultural y la organización de los pueblos indígenas;

- Contribuir a la búsqueda de mejores condiciones de la calidad de vida de los pueblos indígenas.

\section{Objetivos}

El sistema de educación intercultural bilingüe contempla un conjunto de objetivos generales y específicos como se indica a continuación.

\section{Objetivos generales} rales:

Se plantean los siguientes objetivos gene-

- promover la revalorización personal de la población;

- elevar y consolidar la calidad de la educación intercultural bilingüe;

- contribuir al mejoramiento de la calidad de vida de los pueblos indígenas tomando en cuenta sus conocimientos y prácticas sociales;

- recuperar y fortalecer el uso de las distintas lenguas indígenas en todos los ámbitos de la ciencia y la cultura;

- buscar los mecanismos para que las lenguas indígenas sean empleadas en los distintos medios de comunicación;

- fortalecer las formas organizativas propias de los pueblos indígenas;

- fomentar la relación intercultural entre todos los pueblos socio-culturales que conforman el país;

- desarrollar el sistema de educación intercultural bilingüe en función de la realidad socio-cultural, lingüística y económica de la población indígena, así como de sus necesidades y expectativas.

\section{Objetivos específicos}

Se plantean los siguientes objetivos específicos:

- atender las necesidades psicológicas y socio-culturales de la población indígena;

- desarrollar actitudes de investigación en los estudiantes en todos los niveles educativos;

- desarrollar el léxico y la expresión oral y escrita de cada una de las lenguas indígenas; 
- promover la afición, el interés y el gusto por la lectura en la lengua indígena respectiva;

- estimular la escritura como una necesidad de expresión personal y social;

- utilizar las lenguas indígenas como medio de comunicación oral y escrito en todas las áreas del conocimiento;

- incorporar a la educación los conocimientos y características de cada cultura a partir de una investigación sistemática;

- enseñar español como segunda lengua en el contexto de su cultura y de los esquemas mentales propios de la población hispano hablante;

- interrelacionar los diferentes niveles educativos y mantener la cohesión entre los distintos grupos de edad;

- desarrollar propuestas dirigidas a difundir los mecanismos de auto-educación;

- integrar a miembros de las comunidades indígenas en las distintas etapas y actividades del proceso educativo;

- establecer un tipo de currículo que integre los aspectos sicológicos, académicos y sociales necesarios para el desarrollo integral de la persona". ${ }^{3}$

\section{Políticas de gestión institucional de la EIB}

- Descentralización de la EIB

- Gestión técnica y pedagógica

- Fortalecimiento de la lengua y cultura

- Gestión institucional de la DINEIB

- Formación del personal intercultural bilingüe

- Infraestructura con pertinencia cultural

- Rendición de cuentas de la gestión educativa

\section{Principales innovaciones plan- teadas}

Entre las principales innovaciones interculturales que se plantean en este modelo de educación con el fin de abrir un espacio de diálogo epistemológico podemos mencionar las siguientes:

En relación a los objetivos podemos manifestar que, históricamente, la educación ha sido utilizada como una estrategia de dominación y manipulación, mediante la cual la hegemonía se ha mantenido en el poder; en este contexto, por primera vez, los objetivos educativos dirigidos a un sector históricamente aislado se plantean de la siguiente manera.

A. Ofrecer una Educación Intercultural Bilingüe de calidad en todos los niveles y modalidades del sistema, para mejorar el nivel de vida de las nacionalidades y pueblos indígenas y de la sociedad ecuatoriana.

B. Desarrollar programas de formación técnica, profesional, de investigación y extensión en el campo de la ciencia, la tecnología y la cultura de los pueblos originarios y de toda la humanidad.

C. Integrar componentes investigativos, filosóficos y conceptuales con rigurosidad y profundidad en el sistema.

D. Formar profesionales que pongan en práctica los conocimientos de los pueblos y nacionalidades indígenas y sepan aprovechar los recursos y potencialidades locales para impulsar al ser humano a una vida digna, en una sociedad libre y democrática". 4

Las diferentes estrategias como:

-La incorporación en el aprendizaje de las lenguas indígenas, como un proceso de descolonización e interculturalización pedagógico de los niños y niñas desde la educación Infantil Familiar Comunitario que se inicia desde antes de la concepción del niño o niña.

-La incorporación de los maestros que dominen tanto la lengua indígena y el castellano, para que haya una fluida comunicación y aprendizaje con los alumnos, ya que en la educación tradicional se educaba sólo en castellano, res- 
pondiendo a modelos homogenizantes y masificantes con la lengua de dominación que era el castellano, en contra de los postulados de la interculturalidad.

-Rompiendo con la unicidad de las disciplinas, se plantea los estudios de lengua indígena, historia del pueblo indio, literatura andina, ciencias de la vida, la medicina indígena, sumado a los conocimientos universales, la tecnología y por ende el acceso también al inglés como una estrategia de acceso a otros espacios culturales.

-Las escuelas tradicionales se transforman en Centros Educativos Comunitarios- CEC, es decir, una educación no necesariamente dentro del aula con base a los libros que históricamente han estereotipado, como lo demuestra Sebastián Granda en su obra "Textos escolares e interculturalidad en Ecuador"; que toda la comunidad se transforme en una espacio de aprendizaje y de reflexión, con todas sus potencialidades y necesidades, interculturalizando espacios y metodologías de aprendizaje.

-Los talleres de trabajo, las tareas agrícolas, las instituciones, las reuniones, las mingas, las plantas, los animales, los sitios ceremoniales, los ríos, las montañas son espacios y laboratorios de aprendizaje; el espacio físico del CEC debe tener todos los espacios que conforman un hogar, tales como un espacio de trabajo, cocina, animales, un lugar de descanso, de juego, de lectura, de pintura, de música, entre otros.

-Los conocimientos culturales salen de nuestros mayores, quienes por sus años y por su experiencia son considerados grandes sabios en la comunidad y gozan de un gran respeto por ello; muchos no saben leer ni escribir, no tiene mayor poder en estos espacios eurocéntricos de la cultura letrada; pero en la EIB nuestros sabios no son considerados como analfabetos. Aprenden bajo la metodología de "aprender haciendo", con la sabia orientación de sus abuelos, de sus padres, de sus maestros, entre dos sujetos interactuantes, "entre dos sujetos que reflexionan juntos (pueden ser de diferentes culturas) sobre su experiencia y sobre la visión que cada uno tiene del otro. Con ello se generan las condiciones para un pacto de confianza de innegable valor metodológico". 5

- Se trata también de buscar otras racionalidades y otras filosofías como las que se plantean en este sistema educativo. Al respecto Raúl Fornet-Betancourt, al analizar el pensamiento iberoamericano como base de una filosofía intercultural, manifiesta "...se trata de una transformación prospectiva de la filosofía, proyectada hacia el futuro, con la explícita prioridad de fomentar una forma de racionalidad filosófica que sea un proceso de permanente convocación y consultación de racionalidades propias, que en última instancia, resultan ser tales, esto es, se verifican o falsifican como modelos de racionalidad sólo en y mediante ese proceso de diálogo y contraste con las Otras". 6

-El logro de la calidad de educación es el desarrollo de la identidad cultural, desde el cultivo del uso de su lengua indígena y del castellano, la práctica de los valores como la solidaridad, la responsabilidad, el trabajo y el amor a la madre tierra; dentro de un marco de un ser armónico y equilibrado en sentido individual, social y con otras culturas, con miras a la conformación de una sociedad intercultural.

\section{La metodología}

Otro de los planteamientos innovadores es la metodología, que basada en sistema de conocimientos, puede disponer de una serie de recursos intelectuales traducibles en recursos metodológicos utilizables de acuerdo con el desarrollo intelectual de los estudiantes.

Se considera que desde el inicio de aplicación del sistema educativo, se puede recurrir a la utilización de los procesos de reconocimiento, conocimiento, producción y reproducción, y creación y recreación. Los procesos de reproducción y recreación, entendidos, el primero como la posibilidad de apropiarse de conocimientos y transformarlos y, el segundo, como la capacidad 
de partir de los conocimientos adquiridos y desarrollados para llegar a la invención, pueden ser utilizados en etapas más avanzadas del sistema educativo.

El dominio del sistema de conocimiento implica la utilización de los recursos intelectuales como se indica a continuación:

- El reconocimiento utiliza mecanismos de percepción (observación, audicióndegustación, uso del tacto y del olfato), descripción y comparación.

- El conocimiento implica la utilización del pensamiento, la reflexión, el análisis y procesos de diferenciación.

- La producción implica la utilización del conocimiento previo, la definición de opciones y la realización de acciones.

- La reproducción implica el análisis del conocimiento previo, la definición de opciones, la utilización de la imaginación y la ejecución de acciones.

- La creación implica la utilización del conocimiento previo y el uso de la imaginación, el ingenio, la fantasía, los sentimientos.

- La recreación implica la utilización de los conocimientos previos para inventar, a través del descubrimiento de nuevos elementos, del ensayo, la modificación y del empleo de la imaginación, la intuición y la meditación.

- La interpretación implica, además de contar con el conocimiento previo, el uso del análisis y de la reflexión sobre hechos y procesos.

- La planificación implica, junto con el análisis de los conocimientos previos y de la reflexión sobre ellos, la inclusión de procesos de creación en relación con la ubicación del hombre y manejo del mundo en el futuro.

- La recreación implica la utilización de los conocimientos previos para inventar a través del descubrimiento, la imaginación, la intuición, la meditación.

El aprendizaje de las distintas ramas del conocimiento, por su parte, debe tener en cuenta métodos adecuados para los fines que persigue cada área. Son los maestros los que deben conocer determinadas metodologías para poder transmitir los conocimientos del caso, sin que los estudiantes tengan que adquirir conocimientos teóricos que no les compete por encontrarse en otro proceso.

El modelo elimina los mecanismos de dictado, copia o reproducción, así como el sistema de tomar notas cuando se cuenta con el material didáctico necesario, pues lo que se requiere es desarrollar la capacidad de atención y retención.

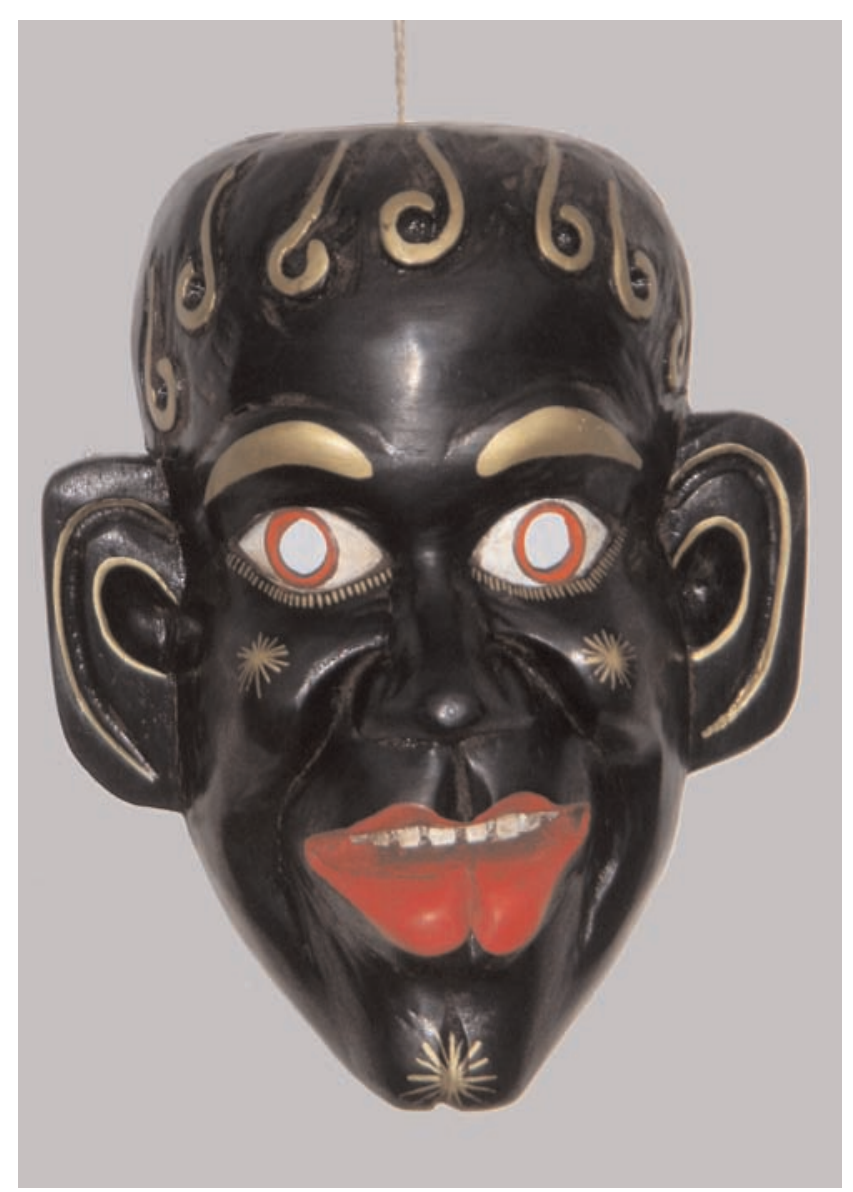


La enseñanza de la lengua nativa se basa en el desarrollo de la expresión oral y escrita de modo que puedan optimizarse la comunicación entre sus hablantes para lo cual se hace innecesario el aprendizaje de teorías gramaticales que no hacen sino confundir a los estudiantes. El desarrollo de la comunicación en la propia lengua implica, además de la práctica oral y escrita, el reconocimiento de los elementos y mecanismos que permiten desarrollar la lengua tanto en relación con el vocabulario como con los conceptos. Implica, además, el reconocimiento y manejo consciente de los elementos paralingüísticos como son el lenguaje corporal, gestual y situacional, así como la producción y creación literarias.

El aprendizaje de español se basa en la metodología de la enseñanza de una segunda lengua que, como en el caso anterior, no requiere el manejo de ninguna teoría gramatical. La metodología debe incluir el aprendizaje de pronunciación -con inclusión de la entonación-, sobre todo en los casos en los que se produce discriminación por esta causa, y del mundo conceptual que caracteriza a la sociedad hispano hablante.

El aprendizaje de español debe eliminar la traducción directa dado que los esquemas mentales que se expresan en las distintas lenguas no son equiparables sino sólo en cierta medida. Para la comprensión de la cultura expresada en la segunda lengua puede recurrirse a un sistema de interpretación que explique con claridad los significados respectivos. Junto con ello se debe incluir el aprendizaje de los elementos paralingüísticos que acompañan las situaciones de comunicación oral y escrita.

La matemática debe tener un tratamiento no teórico sino práctico, de manera que los estudiantes puedan utilizarla en situaciones reales a fin de evitar la multitud de prejuicios a los que se ve sometida la población por desconocer las normas de mercadeo y, en no pocos casos, de explo- tación en la realización de actividades de compra-venta. Además, los conceptos matemáticos básicos deben ser desarrollados a partir de la práctica, por lo que se debe evitar toda memorización anterior a la comprensión de conceptos, siendo un proceso posterior la generalización y abstracción.

Un aspecto que requiere especial atención es el relacionado con las situaciones de trueque y los sistemas monetarios, decimal y otros procedentes de la sociedad externa, y que tienen vigencia universal. En todo caso, se evitará la introducción de métodos destinados a enseñar teorías como es el caso de la gramática estructural o de la teoría de conjuntos.

Para el aprendizaje de historia debe integrarse el concepto de temporalidad, espacio y de sucesión de hechos, es decir, de procesos antes que de situaciones estáticas dadas por la concepción de la historia como la de la presencia de personajes y fechas. La metodología respectiva, sobre todo cuando se trata de la historia universal, debe responder a una concepción activa de la historia y estar ubicada en sus respectivos espacios geopolíticos.

En el área denominada ciencias de la vida se incluye la realización de actividades artísticas y productivas con lo que se integra el conocimiento teórico con la práctica y la estética dando lugar a una metodología activa, centrada en la persona y basada en la teoría integrada de las ciencias. Esta área comprende los conocimientos relacionados con la naturaleza, sus componentes y el actor principal que es el ser humano.

La metodología para la realización de actividades relacionadas con la cultura física y crecimiento personal, se caracteriza por estar encaminada al desarrollo de la persona con todas sus capacidades, a la superación de obstáculos sicológicos debido especialmente a las condiciones producidas por los procesos de dominación y control históricos; al ejercicio lúdico, al aprendizaje de deportes, y al desarrollo y cuidado del cuerpo.

Para tal efecto, se tomará en cuenta la 
situación, la capacidad y el interés de los estudiantes sin que se trate de actividades generalizables sino individualmente aplicables. En la metodología se evitará todo rastro de violencia que pueda resultar perjudicial para los estudiantes $\mathrm{y}$, en todo caso, se cuidará de recurrir a la práctica de ejercicios mecánicos como ha sido la tradición".

\section{Logros alcanzados}

La educación Intercultural Bilingüe es una propuesta de vida, por la vida, en la vida, para la vida de los pueblos y las nacionalidades del Ecuador. Responde al ideal de construir un Estado plurinacional, y una Sociedad intercultural y multilingüe.

\section{Gestión administrativa organizacional}

- La gestión administrativa se genera por intermedio de las 16 direcciones provinciales de educación intercultural bilingüe, 6 direcciones de las nacionalidades y 1 dirección kichwa de la Costa y Galápagos. Se encuentran determinadas 3 direcciones regionales, éstas que se encuentran en proceso de análisis y revisión para su implementación.

- La demanda educativa intercultural bilingüe atiende en los diferentes niveles y modalidades educativas a nivel nacional, de acuerdo con los datos estadísticos descritos a continuación:

- Se ha organizado 58 REDES-CECIBs de los 120 programados para todo el sistema.

\section{Gestión técnica y pedagógica}

- Cuenta con modelo educativo intercultural bilingüe MOSEIB y el modelo de educación infantil familiar comunitario EIFCIB.

- Para la región andina se ha formulado el Rediseño Curricular y el paquete de KUKAYU Pedagógico que se encuentra por producir con el apoyo financiero de MEC y ONGs.

- Se encuentra en ejecución el proyecto EIBAMAZ y EBIZUMA para la región Amazónica.

- Se encuentra en ejecución Programa PLEIB, el programa de la Maestría en

\begin{tabular}{|l|l|r|r|r|}
\hline $\begin{array}{l}\text { NIVELES } \\
\text { MODALIDADES }\end{array}$ & ESTUDIANTES & $\begin{array}{r}\text { ESTABLE- } \\
\text { CIMIENTOS }\end{array}$ & $\begin{array}{c}\text { EDUCA- } \\
\text { DORES }\end{array}$ \\
\hline $\begin{array}{l}\text { EDUCACIÓN } \\
\text { BÁSICA }\end{array}$ & EIFCIB & 7.910 & 363 & 272 \\
\hline BACHILLERATO & Primaria - Secundaria & 103.385 & 2.031 & 5.599 \\
\hline SUPERIOR & Bachillerato & 13.269 & 115 & 1.729 \\
\hline SUBTOTAL EDUCACIÓN REGULAR & 934 & 5 & 173 \\
\hline ALFABETIZACIÓN & Institutos Sup. Pedagógicos & 904 & 6 & 7.601 \\
\hline & Edstitutos Sup. Tecnológicos & $\mathbf{1 1 8 . 4 9 2}$ & 2.157 & 395 \\
\hline CUBTOTAL EDUCACIÓN COMPENSATORIO & 7.587 & 395 & 119 \\
\hline TOTAL GENERAL & Centros Ocupacionales & 1.145 & 240 & 754 \\
\hline
\end{tabular}

Según datos estadísticos de la DINEIB del año 2007. 


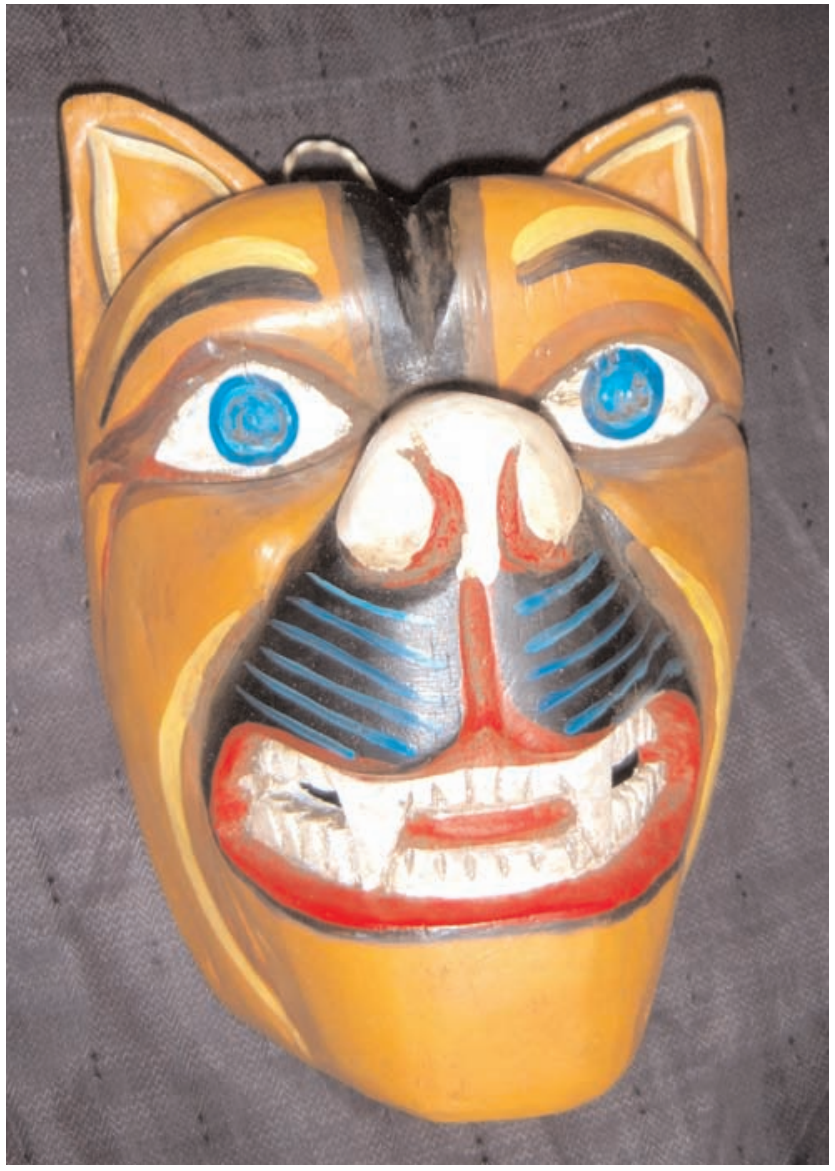

Educación Superior con mención en gestión e investigación.

- Se han elaborado algunos materiales educativos en lenguas indígenas.

- Se ha avanzado en el proceso de normatización y estandarización de las grafías de las lenguas indígenas.

\section{Desarrollo social organizativo}

- Se encuentra organizada la comisión nacional de educación intercultural bilingüe con los representantes de cada una de las nacionalidades.

- La DINEIB se ha constituido en un espacio oculto de unidad de las comunidades, pueblos y nacionalidades indígenas, y en un escenario donde dinamiza los procesos de la interculturalidad.

- La DINEIB, ha formado un cuadro profesional que al momento se encuentra asumiendo diferentes responsabilidades en los organismos estatales, seccionales y no gubernamentales.

- En los colegios y CECs se han formado muchos dirigentes que aportan con su labor social desde diferentes latitudes del país.

- Se ha despertado la conciencia de identidad cultural, por lo que se ha revitalizado los grandes Raymis como el Inti Raymi, Kolla Raymi, Kapak Raymi y Pawkar Raymi en varias nacionalidades indígenas del país.

- Se ha impactado en la sociedad ecuatoriana sobre la revalorización de las lenguas indígenas en especial de la lengua Kichwa como parte de la identidad nacional.

\section{Limitaciones}

- Ataques contra la institucionalidad de la DINEIB de parte del Gobierno y otros organismos.

- Poca conciencia de la razón social de la educación intercultural bilingüe en algunos sectores influyentes y también de los propios actores de la EIB.

- Inmadurez política para designar autoridades y personal docente con responsabilidad en algunos líderes sociales.

- Limitada gestión institucional que involucre la participación de los actores sociales en la toma de decisiones.

- Débil práctica de la interculturalidad que ha reducido a la entrega de cargos burocráticos a personas de otra cultura.

- Carencia de personal docente bilingüe para todas las lenguas indígenas del país.

- Centralismo burocrático de las autoridades de la EIB que no permiten coordinar 
adecuadamente con los técnicos y educadores, como también con las organizaciones indígenas y no indígenas.

- Poca injerencia del gobierno en la orientación y apoyo académico, económico y político.

- Poco apoyo por parte de las organizaciones locales, regionales y nacionales.

- Mala distribución de funciones al personal de las plantas nacional y provinciales.

- Falta de textos y materiales educativos para todas las nacionalidades indígenas del país.

- Falta de toma de conciencia sobre procesos interculturales de la sociedad ecuatoriana.

- Escasa formación de profesionales interculturales por parte de las universidades del país.

- El considerar que la EIB es solamente para indígenas también ha frenado procesos más generalizadores y unificantes.

\section{Conclusiones}

$\mathrm{Al}$ menos en el caso ecuatoriano, en la oficialización de la EIB jugaron un papel decisivo las organizaciones indígenas, los cuáles a través de luchas y movilizaciones lograron concretar sus intereses en 1988; este logro se ve reflejado como parte de los cambios que las sociedades contemporáneas están viviendo, como surgimiento de nuevas alternativas de educar, de vivir y de administrar.
El Modelo de Educación Intercultural Bilingüe en su seno recoge planteamientos muy innovadores, que más allá de caer en juzgamientos infundados es necesario mirar como un espacio de diálogo de saberes, de varias racionalidades y de pueblos diferentes. Para nadie son desconocidos los problemas educativos a nivel general, en este panorama el surgimiento de nuevas y diferentes maneras de acceder al conocimiento merece un cuidadoso apoyo de personas que incursionamos en la noble tarea de educar.

La EIB, en Ecuador, va a cumplir los 20 años de existencia; desde su inicio ha pasado por diferentes etapas: a) fase de experiencias previas hasta 1988, b) fase de oficialización, c) fase de iniciación y estructuración, d) oficialización del Modelo de Educación Intercultural BilingüeMOSEIB, e) fase de profesionalización, f) fase de implementación, g) fase de instrumentalización, h) fase de evaluación (que todavía no se ha planteado).

En estos años se ha hecho presente en todas las comunidades indígenas del país de manera estructural, aunque la aplicación del Modelo Educativo ha sido muy variado, va desde el 30\% a $80 \%$ según el Sistema de Monitoreo SISEMOE, realizado desde la DINEIB, en 2005.

La sociedad ecuatoriana se ha sensibilizado, ha creado espacios de debate y de aprendizajes en los centros educativos, en espacios políticos y organizaciones sociales, y ahora se pregunta si ¿la educación intercultural debe ser sólo para indigenas? o debe ser para todos y todas las que conformamos nuestro país.

1 Plan Estratégico 2007 de la DINEIB.

2 Op. cit.

3 MOSEIB, DINEIB, Quito, 1993.

4 Modelo del Sistema de Educación Intercultural Bilingüe del Ecuador, Quito, 1993.

5 Silvia Rivera, Op. cit., pp. 61.

6 Raúl Fornet-Betancourt. Transformación Intercultural de la Filosofía, EGO, España, 2001, pp. 70.

7 Modelo de Educación Intercultural Bilingüe- DINEIB, Quito, 1993. 


\section{Bibliografía}

DINEIB

1993 Modelo del Sistema de Educación Intercultural Bilingüe-MOSEIB, Quito.

DINEIB

2007 Plan Estratégico de la DINEIB, Quito.

RAÚL FORNET-BETANCOURT

2001 Transformación Intercultural de la Filosofía, EGO, España.
DINEIB

2007 Estadística Nacional de la EIB.

RIVERA, Silvia

Violencias encubiertas en Bolivia. CIPCA ARUWIYIRI.

Ponencias varias. 